\title{
Improved Alignment of Gold Nanorods Embedded in Polymer Films
}

\author{
Stefan Stoenescu, Muthukumaran Packirisamy \\ Dept. of Mechanical Engineering, Concordia University, \\ EV 4.219, 1515 St. Catherine West, Montreal, QC, Canada H3G 2W1 \\ s_stoene@encs.concordia.ca; muthukumaran@sympatico.ca \\ Vo-Van Truong \\ Dept. of Physics, Concordia University, Loyola Science Complex, \\ 7141 Sherbrooke West, Montreal, QC, Canada H4B 1R6 \\ truong.vo-van@concordia.ca
}

\begin{abstract}
The multiple applications of gold nanorods (AuNRs) are most effective if the longitudinal surface plasmon resonance (SPR) can be simultaneously excited in all the involved AuNRs. This can be achieved in well aligned ensembles of AuNRs, obtained by stretching AuNR-polymer composite films, as a possible method. One of the difficulties encountered in applying this method, is the thermal reshaping of the rods, which would change their optical properties. To mitigate this risk, while increasing the plastic deformability of the film, a polymer of high molecular weight mixed with a small amount of plasticizer was chosen as the composite matrix. The resulting composite films could be stretched up to seven times their initial length. To quantify the alignment of the rods, the average orientation angle was calculated based on scanning electron micrographs. An orientational order parameter of 0.92 was found, as compared to 1 , which is the parameter value corresponding to perfect alignment. The anisotropy of the composite film was tested in linearly polarized light.
\end{abstract}

Keywords: aligned gold nanorods, surface plasmon resonance, orientational order parameter, optical anisotropy.

(C) Copyright 2013 Authors - This is an Open Access article published under the Creative Commons Attribution License terms http://creativecommons.org/licenses/by/3.0). Unrestricted use, distribution, and reproduction in any medium are permitted, provided the original work is properly cited.

\section{Introduction}

Metal nanoparticles (NPs) can have their conduction electrons resonantly and coherently excited by the electric field of an incident light beam that provides the exact energy necessary to atomic jump to excited energy states. This phenomenon is known as localized surface plasmon resonance (LSPR), $[1,2]$ as opposed to the propagating surface plasmon resonance (SPR) $[3,4]$ of the conduction electrons at a flat metaldielectric interface. In optically anisotropic nanoparticles (NPs), such as nanorods (NRs), of dimensions much smaller than the wavelength of the incident lightwave, two modes of such resonant oscillation are possible. Depending on their direction of oscillation, these modes can be longitudinal or perpendicular with respect to the longitudinal axis of symmetry of the NRs. Out of the two LSPR modes, the longitudinal one is adjustable over a larger range and is therefore most useful and in demand.

Devices based on ensembles of misaligned rods have averaged out optical properties and are thus characterized by diminished sensitivity to polarized light and function less efficiently. On the other side, devices containing fully aligned rods can reach maximal efficiencies and for this reason, several methods of alignment of the rods have been studied $[5,6,7]$. 
Rod alignment by film stretching is one of these methods. It consists in embedding the AuNRs in a film of thermoplastic polymer that is subsequently heated up to its softening temperature. By simultaneously subjecting the film to tensile stress, a sufficiently large elongation can be obtained. In this process, the nanorods in contact with the polymer chains get entrained and reach a final orientational position parallel with the direction of stretching. It results that the degree of alignment of the rods is directly proportional to the stretch ratio of the film and therefore, the highest possible stretch ratio was attempted in this work. This was achieved by slightly modifying the composition of the composite matrix in order to improve its plastic deformability. A stretching device was also designed in order to reduce the risk of film fracture. Finally, the alignment of the AuNRs was assessed qualitatively by scanning electron microscopy (SEM) and quantified by calculating an average orientation angle and an orientational order parameter.

\section{Improving the Plastic Deformability}

The matrix of this type of composite film is usually chosen to consist of polyvinyl alcohol (PVA) because this polymer can be drawn into thin and transparent films. This is due to its thermoplastic properties, which makes that it softens when heated up to its glass transition temperature $\mathrm{T}_{\mathrm{g}}\left(85^{\circ} \mathrm{C}\right)$ and flows under tensile stress. If PVA is cooled back to room temperature, it regains its hardness in a reversible cycle. This is possible because its molecules consist of many thousands of carbon atoms bound together by single and strong covalent bonds, which form long linear chains with rotational, bending and twisting flexibility in three dimensions [8]. Therefore, a wellstirred PVA aqueous solution with well dispersed AuNRs may reach a highly entangled state of its chains. If the mixture is allowed to dry, it will solidify in this state, forming a film. By heating up the film while simultaneously applying a slowly increasing stretching force, the secondary bonding forces between chains will be broken, allowing them to disentangle. Eventually, the polymer chains will be straightened out with their axes oriented along the stretching direction and the rods aligned parallelly to the stretch direction, as well.

Thus, matrix polymers of longer molecular chains allow for higher film stretch ratios because the relative displacement of the chains is possible along longer distances. However, the higher molecular weights of such polymers also have higher glass transition temperature. But heating up to this temperature is limited by the risk of thermal reshaping of the rods with altered optical properties as a consequence [1, 9]. In order to depress the softening temperature, a small amount of glycerol as plasticizer was mixed in the polymer solution. The selection of glycerol was based on a study [10] that found that it was one the plasticizers most compatible with PVA.

Subsequently, the transparency of the glycerol-PVA films was tested in the visible-NIR range and it was found to have been well maintained. This verification was necessary in order to insure that the optical excitation of the embedded AuNRs would not be obstructed.

Finally, films with glycerol of $2 \%$ and $4 \%$ mass percent concentration of PVA were tested and all could be stretched up to a ratio of seven. However, because one undesired side effect of the plasticizer is that the solidified film is less rigid, the optimal concentration of glycerol was chosen such as to insure that the resulting film could maintain a flat enough surface over a long period of time, after stretching.

Previous reports have indicated that a stretch ratio of four to six would be sufficient for a perfect alignment [11]. In order to insure the best possible alignment of AuNRs embedded in the composite film, we aimed in this work for a stretch ratio of seven.

\section{Experimental}

Completely hydrolyzed PVA 146,000-186,000 MW, glycerol G9012 ( $\geq 99.50 \%$ ) and gold nanorods 716812 (diam. $\times$ L $10 \mathrm{~nm} \times 38 \mathrm{~nm}+/-10 \%$ ) were all purchased from Sigma-Aldrich and used as received. A volume of $11 \mathrm{ml}$ of AuNR solution of concentration $35 \mu \mathrm{g} / \mathrm{ml}$ was used for forming of a composite film.

A mass of $2.04 \mathrm{~g}$ PVA granules was gradually mixed in $20 \mathrm{ml}$ distilled and deionized water and slowly heated up to about $95^{\circ} \mathrm{C}$ under stirring, until a clear and transparent $10 \%$ PVA solution was obtained. After the solution cooled down to about $35^{\circ} \mathrm{C}, 32 \mu \mathrm{l}$ glycerol $(2 \%$ of the PVA mass), was dropped into the PVA solution under stirring for an additional 30 minutes. The solution was subsequently degassed. Finally, $3.0 \mathrm{~g}$ of the above PVA-glycerol water solution was poured into a 50 $\mathrm{ml}$ beaker and $11 \mathrm{ml}$ of the $35 \mu \mathrm{g} / \mathrm{ml}$ AuNRs colloidal solution was added, well stirred and sonicated for about 30 minutes. The AuNR-PVA solution was subsequently cast into a $60 \times 60 \mathrm{~mm}^{2}$ flat mold and allowed to solidify for $48 \mathrm{hrs}$ at room temperature. The film was afterwards kept in an oven at $40^{\circ} \mathrm{C}$ temperature for 24 
hrs to make sure it was completely dry. After having peeled it off, the film was cut into 20 by $30 \mathrm{~mm}$ rectangular strips. The strips were then clamped into the stretching device and gradually stretched under heating at about $65^{\circ} \mathrm{C}$.

Absorbance spectra were obtained using a Perkin Lambda 650 spectrophotometer, in the visible - NIR region. Micrographs were taken with the JEOL JSM840 scanning electron microscope.

\subsection{Morphological Characterization}

The average orientation angle of the rod ensemble contained in a film strip was calculated based on dimensional data of more than 160 rods collected from several SEM micrographs. We show one such micrograph in the panel (a) of Figure 1 . In order to define the orientational angle of the rods, we show a few typical ones schematically depicted by golden rectangles, randomly oriented and zoomed-up in Figure 1.

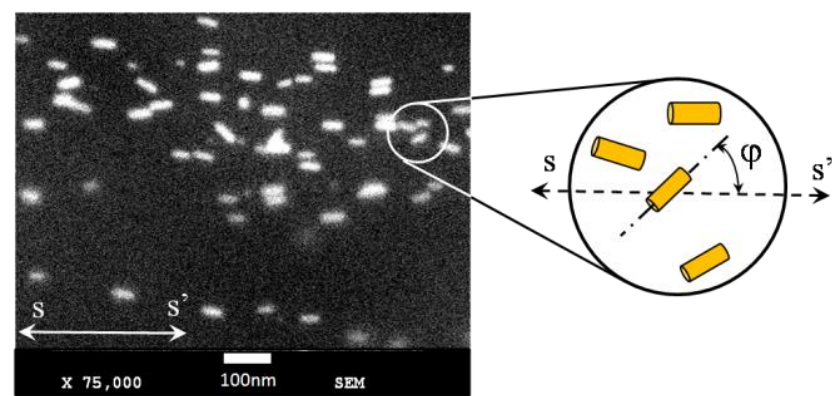

Figure 1. SEM micrograph of a AuNR-PVA composite film showing rods more or less aligned along the stretch direction $\mathrm{s}-\mathrm{s}^{\prime}$. The inset drawing presents a detailed view of the rods and the definition of the orientational angle $\varphi$ of a typical rod with respect to the direction $s-s^{\prime}$.

The stretch ratio " $R_{s}$ " was defined as the ratio: $R_{s}=$ $\mathrm{L}_{\mathrm{f}} / \mathrm{L}_{0}$ where we denoted the initial and final length, by $\mathrm{L}_{0}$ and $\mathrm{L}_{\mathrm{f}}$, respectively. The film strip is stretched along the $s$ - s' direction, represented by the double headed arrow. The longitudinal elongation is associated with a transversal contraction, caused by the Poisson effect. The orientation of the rods was measured by the angle " $\varphi$ ", defined between the long axis of the rod and the stretch direction $s-s^{\prime}$, as indicated in Figure 1.

The resulting sample mean was of $\varphi_{\text {avg }}=3.5^{\circ}$ with a standard deviation of $\sigma=11.05^{\circ}$.

A two-dimensional orientational order parameter defined as $S=2<\cos ^{2} \varphi>-1[6,12]$ was found with a value of $S=0.92$. This compares well to the value of $S=$
1 , which is the value of the parameter corresponding to the perfect alignment of the rods.

\subsection{Optical Characterization}

A plane polarized lightwave with the electric field parallel $\left(E_{\|}\right)$with the stretch direction s'-s incident on the composite film, is strongly absorbed at the longitudinal wavelength and a minimal radiation energy is allowed to pass through the film, as seen in panel (a) of Figure 2. If the electric field of the lightwave is perpendicular $\left(E_{\perp}\right)$ to $\mathrm{s}-\mathrm{s}^{\prime}$, then the lightwave energy is transmitted unabated through the film. Therefore, the composite film has the same optical properties as a dichroic polarizer with the transmission axis perpendicular to the stretch direction.
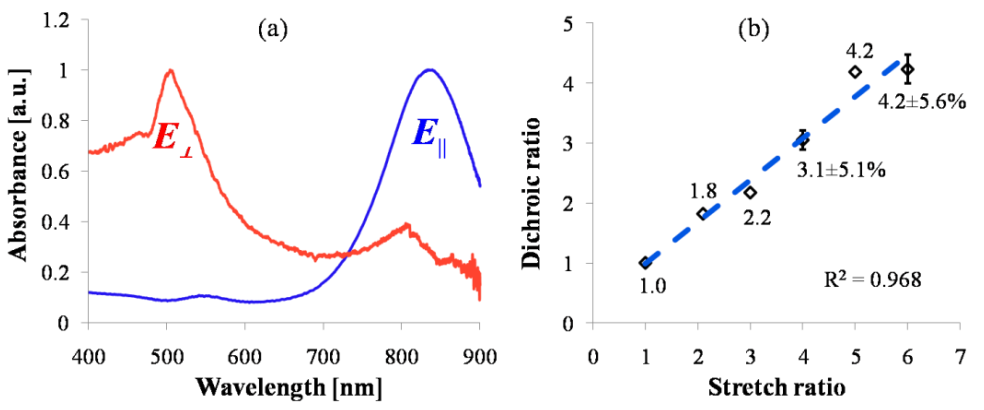

Figure 2. (a) Normalized absorbance spectra of uniaxially tretched AuNR-PVA film illuminated by plane polarized light with the electric field parallel $\left(\mathrm{E}_{\|}\right)$and perpendicular $\left(E_{\perp}\right)$ to

the s' - s direction. (b) Linear trend of the dichroic ratio dependence on the stretch ratio.

The above statement holds for the ideal case of perfectly aligned AuNRs embedded in a composite film. In reality however, a small absorbance peak can still be noticed located at the wavelength of the transverse SPR, in panel (a), for light polarized parallel with the stretch direction. Similarly, a small absorbance peak is seen to persist at the longitudinal SPR wavelength, for light polarized perpendicular to the stretch direction. This demonstrates the existence of a large enough number of AuNRs that could not be aligned during the stretching process. These peaks would not exist in a perfectly aligned ensemble of rods.

The polarization performance of the composite film can be characterized by the dichroic ratio, defined as the ratio of the absorbance intensities for plane polarized light with the electric field parallel and perpendicular to s'- s. The dependence of the dichroic ratio on the stretch ratio is shown in panel (b) of Figure 2. The trend line fitted to the experimental data 
suggests that the dichroic ratio depends linearly on the stretch ratio.

\section{Conclusions}

In this work, a thermoplastic polymer (PVA) of high molecular weight, mixed with a small amount of plasticizer (glycerol) was used as matrix material for making AuNRs - composite films. Using a stretching device designed to avoid factors promoting the fracture of the composite film, a stretch ratio of seven was possible.

The resulting alignment of the AuNRs was quantified by calculating an average orientational angle of $3.5^{\circ}$ and a two-dimensional orientational order parameter of $S=0.92$, which is $8 \%$ off the value of a perfectly aligned ensemble.

The AuNRs-PVA composite film was shown to behave like a dichroic polarizer with the transmission axis perpendicular to the stretch direction. A trendline fitted to the measured dichroic ratio suggests a linear proportionality between its dichroic ratio and the stretch ratio.

The dichroic properties of the film can be useful in applications for imaging, nonlinear optics, thermal therapy or data encoding.

\section{Acknowledgements}

We wish to warmly thank Nicole A. MacDonald, physicist with Le Centre de Caractérisation Microscopique des Matériaux, Montreal, for her skillful and dedicated efforts in taking the SEM images of the embedded nanorods.

We also wish to thank the Engineering Design and Manufacturing Laboratories of the Mechanical and Industrial Engineering department at Concordia University, Montreal, for the professional advice and fabrication of the stretching device.

\section{References}

[1] J. Perez-Juste, B. Rodriguez-Gonzalez, P. Mulvaney, L.M. Liz-Marzan "Optical control and patterning of gold-nanorods-poly(vinyl alcohol) films", Advanced Functional Materials, 2005, 15, 1065-1071.

[2] K.A. Willets and R. P. Van Duyne "Localized Surface Plasmon Resonance Spectroscopy and Sensing", Annual Reviews of Physical Chemistry, 2007, 8:26797.
[3] C. F. Bohren, D.R. Huffman "Absorption and Scattering of Light by Small Particles", 1983, John Wiley \& Sons, Inc.

[4] U. Kreibig, M. Vollmer "Optical properties of metal clusters", 1995, Springer-Verlag Berlin Heidelberg.

[5] B. M. I. van der Zande, G.J.M Koper and H.N.W Lekkerkerker "Alignment of Rod-Shaped Gold Particles by Electric Fields", Journal of Physical Chemistry. B, 1999, 103, 5754-5760.

[6] Z. Hu, M.D. Fischbein, C. Guerner and M. Drndic "Electric-Field-Driven Accumulation and Alignment of CdSe and CdTe Nanorods in Nanoscale Devices", Nano Letters, 2006, 6, 11, 2585-2591.

[7] A.J. Mieszawska, G.W. Slawinski and F.P. Zamborini "Directing the Growth of Highly Aligned Gold Nanorods through a Surface Chemical Amidation Reaction" Journal of the American Chemical Society, 2006, 128, 5622-5623.

[8] W.D. Callister, D.G. Rethwisch "Materials Science and Engineering - An Introduction", 2010, John Wiley \& Sons, Inc.

[9] Y. Liu, E.N. Mills, R.J. Composto "Tuning optical properties of gold nanorods in polymer films through thermal reshaping" Journal of Materials Chemistry, 2009, 19, 2704-2709.

[10] C.A. Finch "Polyvinyl alcohol, properties and applications" Journal of Polymer Science: Polymer Letters Edition, 2003, 12, 2, 105-106, Wiley, New York.

[11] B.M.I. van der Zande, L. Pages, R.A.M. Hikmet, A. van Blaaderen, "Optical Properties of Aligned RodShaped Gold Particles Dispersed in Poly(vinyl alcohol) Films", Journal of Physical Chemistry B, 1999, 103, 5761-5767.

[12] W. Ahmed, E.S. Kooij, A. van Silfhout and B. Poelsema "Quantitative Analysis of Gold Nanorod Alignment after Electric Field-Assisted Deposition", Nano Letters, 2009, 9, 11, 3786-3794. 